\title{
Cluster-tilted algebras as trivial extensions
}

\author{
I. Assem, T. Brüstle and R. Schiffler*
}

\begin{abstract}
Given a finite dimensional algebra $C$ (over an algebraically closed field) of global dimension at most two, we define its relation-extension algebra to be the trivial extension $C \ltimes \operatorname{Ext}_{C}^{2}(D C, C)$ of $C$ by the $C$ - $C$-bimodule $\operatorname{Ext}_{C}^{2}(D C, C)$. We give a construction for the quiver of the relationextension algebra in case the quiver of $C$ has no oriented cycles. Our main result says that an algebra $\tilde{C}$ is cluster-tilted if and only if there exists a tilted algebra $C$ such that $\tilde{C}$ is isomorphic to the relation-extension of $C$.
\end{abstract}

\section{Introduction}

Cluster categories were introduced in [6], and, for type $A_{n}$ also in [12], as a means for a better understanding of the cluster algebras of Fomin and Zelevinsky 14, 15]. They are defined as follows: let $A$ be a hereditary algebra, and $\mathcal{D}^{b}(\bmod A)$ be the derived category of bounded complexes of finitely generated $A$-modules, then the cluster category $\mathcal{C}_{A}$ is the orbit category of $\mathcal{D}^{b}(\bmod A)$ under the action of the functor $F=\tau^{-1}$ [1], where $\tau$ is the Auslander-Reiten translation in $\mathcal{D}^{b}(\bmod A)$ and $[1]$ is the shift.

In [7, Buan, Marsh and Reiten defined the cluster-tilted algebras as follows. Let $A$ be a hereditary algebra, and $\tilde{T}$ be a tilting object in $\mathcal{C}_{A}$, that is, an object such that $\operatorname{Ext}_{\mathcal{C}_{A}}^{1}(\tilde{T}, \tilde{T})=0$ and the number of isomorphism classes of indecomposable summands of $\tilde{T}$ equals the number of isomorphism classes of simple $A$-modules. Then the endomorphism algebra $\operatorname{End}_{\mathcal{C}_{A}}(\tilde{T})$ is called clustertilted. Since then, these algebras have been the subject of many investigations, see, for instance, 17, 8, 9, 10, 11, 12, 13, 21. In several particular cases, it was shown that the quiver of a cluster-tilted algebra was obtained from that of a tilted algebra by replacing relations by arrows, see, for instance [10, 11]. Our objective in this paper is to prove this statement in a more general context (not depending on the representation type). This is achieved by looking at clustertilted algebras as trivial extensions of tilted algebras by a bimodule which we explicitely describe (compare [3]).

${ }^{*}$ The first and the second author gratefully acknowledge partial support form the NSERC of Canada. The second author also thanks the universities of Sherbrooke and Bishop's for partial support. The third author was partially supported by the University of Massachusetts. 
For this purpose, we let $C$ be a finite dimensional algebra of global dimension two (over an algebraically closed field), and consider the $C$-C-bimodule $\operatorname{Ext}_{C}^{2}(D C, C)$ with the natural action. The trivial extension $C \ltimes \operatorname{Ext}_{C}^{2}(D C, C)$ is called the relation-extension algebra of $C$. Our first main result (Theorem 2.6) describes the quiver of the relation-extension of $C$ in the case where the quiver of $C$ has no oriented cycles: we prove that indeed this quiver is given by replacing each element in a (minimal) system of relations by an arrow (going in the opposite direction to the relation). We then prove the main result of this paper.

Theorem 1.1 An algebra $\tilde{C}$ is cluster-tilted if and only if there exists a tilted algebra $C$ such that $\tilde{C}$ is the relation-extension of $C$.

We note that several tilted algebras may correspond to the same clustertilted algebra, so this mapping is not bijective. On the other hand, there clearly exist relation-extension algebras which are not cluster-tilted.

Combining the above theorem with Theorem 2.6 we deduce the construction of the quiver of a cluster-tilted algebra. This allows, for instance, as done in [11, to relate the list of tame concealed algebras of Happel and Vossieck [18] with Seven's list of minimal infinite cluster quivers [23].

This paper consists of two sections. The first one describes relation-extension algebras and their quivers, and the second is devoted to the cluster-tilted algebras. Moreover, we give several examples.

Th. Brüstle wishes to thank Claus Michael Ringel and Idun Reiten for interesting discussions on this problem.

\section{Relation-extension algebras}

\subsection{The definition}

Throughout this paper, algebras are basic and connected finite dimensional algebras over a fixed algebraically closed field $k$. For an algebra $C$, we denote by $\bmod C$ the category of finitely generated right $C$-modules and by $\mathcal{D}^{b}(\bmod C)$ the derived category of bounded complexes over $\bmod C$. The functor $D=$ $\operatorname{Hom}_{k}(-, k)$ is the standard duality between $\bmod C$ and $\bmod C^{o p}$. For facts about $\bmod C$ or $\mathcal{D}^{b}(\bmod C)$, we refer to $2,22,17$.

Let $C$ be an algebra. We recall that the trivial extension of $C$ by a $C-C$ bimodule $M$ is the algebra $C \ltimes M$ with underlying $k$-vector space

$$
C \oplus M=\{(c, m) \mid c \in C, m \in M\}
$$

and the multiplication defined by

$$
(c, m)\left(c^{\prime}, m^{\prime}\right)=\left(c c^{\prime}, c m^{\prime}+m c^{\prime}\right)
$$

for $c, c^{\prime} \in C$ and $m, m^{\prime} \in M$. For trivial extension algebras, we refer to [16, 1].

In this section, we introduce a particular class of trivial extension algebras which are useful for studying the cluster-tilted algebras. 
Definition 2.1 Let $C$ be a finite dimensional algebra of global dimension at most two, and consider the $C$-C-bimodule $\operatorname{Ext}_{C}^{2}(D C, C)$ (with the natural action). The trivial extension

$$
C \ltimes \operatorname{Ext}_{C}^{2}(D C, C)
$$

is called the relation-extension of $C$.

Clearly, any hereditary algebra is (trivially) the relation-extension of itself. On the other hand, if $C$ is of global dimension equal to two (thus not hereditary) there exist two simple $C$-modules $S$ and $S^{\prime}$ such that $\operatorname{Ext}_{C}^{2}\left(S, S^{\prime}\right) \neq 0$. Denoting by $I$ the injective envelope of $S$ and by $P^{\prime}$ the projective cover of $S^{\prime}$, the short exact sequences

$$
\begin{gathered}
0 \longrightarrow S \longrightarrow I \longrightarrow I / S \longrightarrow 0 \\
\text { and } 0 \longrightarrow \operatorname{rad} P^{\prime} \longrightarrow P^{\prime} \longrightarrow S^{\prime} \longrightarrow 0
\end{gathered}
$$

induce an epimorphism $\operatorname{Ext}_{C}^{2}\left(I, P^{\prime}\right) \rightarrow \operatorname{Ext}_{C}^{2}\left(S, S^{\prime}\right)$. Thus $\operatorname{Ext}_{C}^{2}\left(I, P^{\prime}\right) \neq 0$ and consequently $\operatorname{Ext}_{C}^{2}(D C, C) \neq 0$.

\section{$2.2 \quad$ A system of relations}

We wish to describe the bound quiver of a relation-extension algebra. Let $C$ be an algebra. It is well-known that there exists a (uniquely determined) quiver $Q_{C}$ and an admissible ideal $I$ of the path algebra $k Q_{C}$ of $Q_{C}$ such that $C \cong k Q_{C} / I$, see, for instance, [5]. We denote by $\left(Q_{C}\right)_{0}$ the set of points of $Q_{C}$ and by $\left(Q_{C}\right)_{1}$ its set of arrows. For each point $x \in\left(Q_{C}\right)_{0}$, we let $e_{x}$ denote the corresponding primitive idempotent of $C$, and by $S_{x}, P_{x}, I_{x}$ respectively, the corresponding simple, indecomposable projective and indecomposable injective $C$-module.

Following [4, we define a system of relations for $C \cong k Q_{C} / I$ to be a subset $R$ of $\bigcup_{x, y \in\left(Q_{C}\right)_{0}} e_{x} I e_{y}$ such that $R$, but no proper subset of $R$, generates $I$ as a two-sided ideal of $k Q_{C}$. Thus, for any $x, y \in\left(Q_{C}\right)_{0}$, the elements of $R \cap\left(e_{x} I e_{y}\right)$ are linear combinations of paths (of length at least two) from $x$ to $y$. We need the following result.

Lemma $2.2(([4,1.2]))$ Let $C \cong k Q_{C} / I$ be such that $Q_{C}$ has no oriented cycles and $R$ be a system of relations for $C$. Then, for each $x, y \in\left(Q_{C}\right)_{0}$, the cardinality of the set $R \cap\left(e_{x} I e_{y}\right)$ is independent of the chosen system of relations for $C$, and equals $\operatorname{dim}_{k} \operatorname{Ext}_{C}^{2}\left(S_{x}, S_{y}\right)$.

\subsection{The quiver of a trivial extension}

We start with the following easy lemma.

Lemma 2.3 Let $C$ be an algebra, and $M$ be a $C$-C-bimodule. The quiver $Q_{C \ltimes M}$ of the trivial extension of $C$ by $M$ is constructed as follows:

1. $\left(Q_{C \ltimes M}\right)_{0}=\left(Q_{C}\right)_{0}$ 
2. For $x, y \in\left(Q_{C}\right)_{0}$, the set of arrows in $Q_{C \ltimes M}$ from $x$ to $y$ equals the set of arrows in $Q_{C}$ from $x$ to $y$ plus

$$
\operatorname{dim}_{k} \frac{e_{x} M e_{y}}{e_{x} M(\operatorname{rad} C) e_{y}+e_{x}(\operatorname{rad} C) M e_{y}}
$$

additional arrows from $x$ to $y$.

Proof. Since $M \subset \operatorname{rad}(C \ltimes M)$, the quivers of $C \ltimes M$ and of $C$ have the same points. The arrows in the quiver of $C \ltimes M$ correspond to a $k$-basis of the vector space

$$
\operatorname{rad}(C \ltimes M) / \operatorname{rad}^{2}(C \ltimes M) .
$$

Now, as a vector space

$$
\operatorname{rad}(C \ltimes M)=\operatorname{rad} C \oplus M
$$

and since $M^{2}=0$ in $C \ltimes M$,

$$
\operatorname{rad}^{2}(C \ltimes M)=\operatorname{rad}^{2} C \oplus[M(\operatorname{rad} C)+(\operatorname{rad} C) M] .
$$

Since $\operatorname{rad}^{2} C \subset \operatorname{rad} C$ and $M(\operatorname{rad} C)+(\operatorname{rad} C) M \subset M$ and since the arrows of $Q_{C}$ correspond to a basis of $\operatorname{rad} C / \operatorname{rad}^{2} C$, the additional arrows of $Q_{C \ltimes M}$ correspond to a $k$-basis of $M /[M(\operatorname{rad} C)+(\operatorname{rad} C) M]$. The arrows from $x$ to $y$ are obtained upon multiplying by $e_{x}$ on the left and by $e_{y}$ on the right.

\subsection{The top of $\operatorname{Ext}_{C}^{2}(D C, C)$}

In the situation of section 2.3 the $C$ - $C$-bimodule $M(\operatorname{rad} C)+(\operatorname{rad} C) M$ is the radical of $M$, and the quotient $M /[M(\operatorname{rad} C)+(\operatorname{rad} C) M]$ is its top. In the case of relation-extension algebras, we are interested in the top of $\operatorname{Ext}_{C}^{2}(D C, C)$.

Lemma 2.4 Let $C$ be an algebra of global dimension two. The top of the $C-C$ bimodule $\operatorname{Ext}_{C}^{2}(D C, C)$ is isomorphic to $\operatorname{Ext}_{C}^{2}(\operatorname{soc} D C$, top $C)$.

Proof. The short exact sequences

$$
\begin{aligned}
& 0 \longrightarrow \operatorname{rad} C \stackrel{i}{\longrightarrow} C \longrightarrow \text { top } C \longrightarrow 0 \\
& 0 \longrightarrow \operatorname{soc} D C \stackrel{j}{\longrightarrow} D C \longrightarrow D C / \operatorname{soc} D C \longrightarrow 0
\end{aligned}
$$

where $i, j$ are the inclusions, induce a commutative diagram with exact rows and columns (the zeros are obtained from the condition that the global dimension 
of $C$ is two).

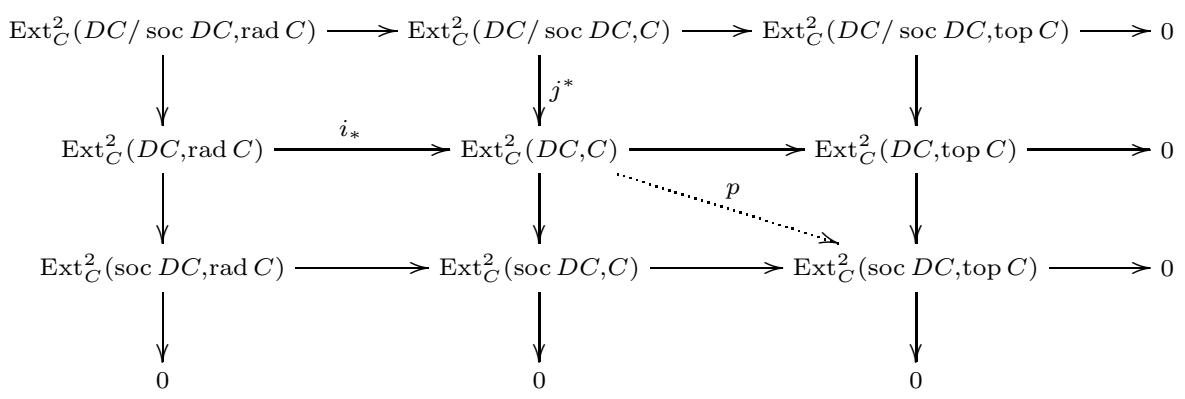

By the commutativity of the lower-right square, there exists an epimorphism $p: \operatorname{Ext}_{C}^{2}(D C, C) \rightarrow \operatorname{Ext}_{C}^{2}(\operatorname{soc} D C$, top $C)$. We thus only need to show that the kernel of $p$ is isomorphic to the radical

$$
\operatorname{Ext}_{C}^{2}(D C, C)(\operatorname{rad} C)+(\operatorname{rad} C) \operatorname{Ext}_{C}^{2}(D C, C)
$$

of the $C$ - $C$-bimodule $\operatorname{Ext}_{C}^{2}(D C, C)$. Now an easy diagram chasing yields

$$
\operatorname{Ker} p=\operatorname{Im} j^{*}+\operatorname{Im} i_{*} .
$$

Thus, it suffices to prove that

$$
\operatorname{Im} i_{*}=(\operatorname{rad} C) \operatorname{Ext}_{C}^{2}(D C, C) \quad \text { and } \quad \operatorname{Im} j^{*}=\operatorname{Ext}_{C}^{2}(D C, C)(\operatorname{rad} C) .
$$

We only show the first equality, the second is shown similarly. Let

$$
0 \longrightarrow P_{2} \stackrel{d_{2}}{\longrightarrow} P_{1} \stackrel{d_{1}}{\longrightarrow} P_{0} \stackrel{d_{0}}{\longrightarrow} D C \longrightarrow 0
$$

be a projective resolution of $D C$. By definition

$$
\operatorname{Ext}_{C}^{2}(D C, C)=\operatorname{Hom}_{C}\left(P_{2}, C\right) / \operatorname{Im}_{\operatorname{Hom}}\left(d_{2}, C\right) .
$$

We first claim that the image of the map

$$
i_{0}=\operatorname{Hom}_{C}\left(P_{2}, i\right): \operatorname{Hom}_{C}\left(P_{2}, \operatorname{rad} C\right) \rightarrow \operatorname{Hom}_{C}\left(P_{2}, C\right)
$$

is equal to $(\operatorname{rad} C) \operatorname{Hom}_{C}\left(P_{2}, C\right)$. Indeed, the product $r f$ with $r \in \operatorname{rad} C$ and $f \in \operatorname{Hom}_{C}\left(P_{2}, C\right)$ is easily seen to factor through $\operatorname{rad} C$. Therfore, we have $(\operatorname{rad} C) \operatorname{Hom}_{C}\left(P_{2}, C\right) \subset \operatorname{Im} i_{0}$. On the other hand, there is an isomorphism of $k$-vector spaces

$$
(\operatorname{rad} C) \operatorname{Hom}_{C}\left(P_{2}, C\right) \cong \operatorname{Hom}_{C}\left(P_{2}, \operatorname{rad} C\right) .
$$

Since $i_{0}$ is injective, this establishes our claim.

Now, the image of $i_{*}$ is generated by the residual classes (modulo the image of $\left.\operatorname{Hom}_{C}\left(d_{2}, C\right)\right)$ of the products $i g$, with $g \in \operatorname{Hom}_{C}\left(P_{2}, \operatorname{rad} C\right)$. These are the residual classes of the elements in $\operatorname{Im} i_{0}$ thus, by our claim above, the residual classes of the elements of the form $r f$ with $r \in \operatorname{rad} C$ and $f \in \operatorname{Hom}_{C}\left(P_{2}, C\right)$. We deduce that $\operatorname{Im} i_{*}=(\operatorname{rad} C) \operatorname{Ext}_{C}^{2}(D C, C)$, as required. 
Remark 2.5 The proof of this lemma can easily be generalised to show that, for an algebra $C$ of global dimension at most $m$, the top of the bimodule $\operatorname{Ext}_{C}^{m}(D C, C)$ is equal to $\operatorname{Ext}_{C}^{m}(\operatorname{soc} D C$, top $C)$.

\subsection{The quiver of a relation-extension}

The following theorem states that the quiver of the relation-extension algebra is obtained from the quiver of the original algebra by adding, for each pair of points $x, y$, one arrow from $x$ to $y$ for each relation from $y$ to $x$. This justifies the name "relation-extension".

Theorem 2.6 Let $C \cong k Q_{C} / I$ be an algebra of global dimension at most two, such that $Q_{C}$ has no oriented cycles, and let $R$ be a system of relations for $C$. The quiver of the relation-extension algebra $C \ltimes \operatorname{Ext}_{C}^{2}(D C, C)$ is constructed as follows:

(a) $\left(Q_{C \ltimes \operatorname{Ext}_{C}^{2}(D C, C)}\right)_{0}=\left(Q_{C}\right)_{0}$

(b) For $x, y \in\left(Q_{C}\right)_{0}$, the set of arrows in $Q_{C \ltimes \mathrm{Ext}_{C}^{2}(D C, C)}$ from $x$ to $y$ equals the set of arrows in $Q_{C}$ from $x$ to $y$ plus $\operatorname{Card}\left(R \cap\left(e_{y} I e_{x}\right)\right)$ additional arrows.

Proof. Let $S_{1}, S_{2}, \ldots, S_{n}$ denote a complete set of representatives of the isomorphism classes of simple $C$-modules, and set $S=\oplus_{i=1}^{n} S_{i}$. Since $C$ is basic, the module $S$ is isomorphic to the top of $C$ and to the socle of $D C$. By Lemma 2.2. the relations of $R$ correspond to a $k$-basis of $\operatorname{Ext}_{C}^{2}(S, S)$. By Lemma 2.4 the $C$-C-bimodule $\operatorname{Ext}_{C}^{2}(S, S)$ is isomorphic to the top of $\operatorname{Ext}_{C}^{2}(D C, C)$. Lemma 2.3 then implies that the number of additional arrows from $x$ to $y$ equals the $k$-dimension of the vector space $e_{x} \operatorname{Ext}_{C}^{2}(S, S) e_{y}=\operatorname{Ext}_{C}^{2}\left(S_{y}, S_{x}\right)$, and the result follows.

In particular, the quiver of a non-hereditary relation-extension algebra always contains oriented cycles.

\subsection{The indecomposable projectives}

It would be useful to know a system of relations for the relation-extension algebra $C \ltimes \operatorname{Ext}_{C}^{2}(D C, C)$ starting from one for $C$. In actual examples, such a system is easily obtained once we know the indecomposable projective modules. In order to state the next lemma, we need a notation: for each $x \in\left(Q_{C}\right)_{0}$, we denote by $\tilde{P}_{x}$ the corresponding indecomposable projective $C \ltimes \operatorname{Ext}_{C}^{2}(D C, C)$-module. Also, we note that $C$-modules can always be considered as $C \ltimes \operatorname{Ext}_{C}^{2}(D C, C)$ modules under the standard embedding.

Lemma 2.7 Let $C$ be an algebra of global dimension at most two. Then, for each $x \in\left(Q_{C}\right)_{0}$, we have a short exact sequence in $\bmod \left(C \ltimes \operatorname{Ext}_{C}^{2}(D C, C)\right)$

$$
0 \rightarrow \operatorname{Ext}_{C}^{2}\left(D C, P_{x}\right) \rightarrow \tilde{P}_{x} \stackrel{p_{x}}{\rightarrow} P_{x} \rightarrow 0
$$

where $p_{x}$ is a projective cover. 
Proof. Since both $P_{x}$ and $\tilde{P}_{x}$ admit $S_{x}$ as a simple top, there indeed exists a projective cover morphism $p_{x}: \tilde{P}_{x} \rightarrow P_{x}$. On the other hand, $\operatorname{Ext}_{C}^{2}\left(D C, P_{x}\right) \cong$ $e_{x} \operatorname{Ext}_{C}^{2}(D C, C)$ is clearly a submodule of the $C \ltimes \operatorname{Ext}_{C}^{2}(D C, C)$-module $\tilde{P}_{x}$. The result then follows from the isomorphism of $k$-vector spaces

$$
\tilde{P}_{x}=e_{x}\left(C \ltimes \operatorname{Ext}_{C}^{2}(D C, C)\right) \cong P_{x} \oplus \operatorname{Ext}_{C}^{2}\left(D C, P_{x}\right) .
$$

\subsection{An example}

Example 2.8 Let $C$ be given by the quiver

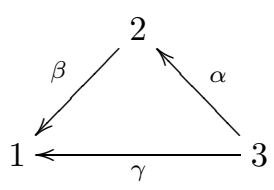

bound by the relation $\alpha \beta=0$. Thus

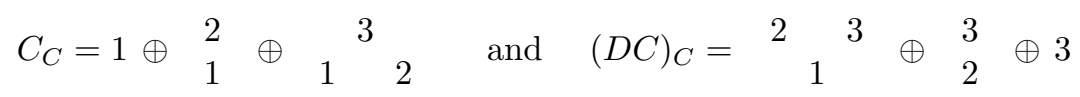

where the indecomposable projectives and injectives are represented by their Loewy series. It is easily seen that the global dimension of $C$ is two. By Theorem [2.6. the quiver of $C \ltimes \operatorname{Ext}_{C}^{2}(D C, C)$ is obtained by adding to $Q_{C}$ a single arrow $\delta: 1 \rightarrow 3$.

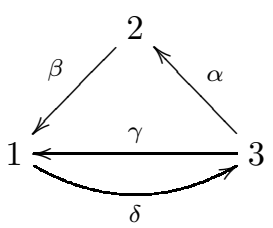

We now compute the new indecomposable projective modules. A simple calculation yields

$$
\begin{aligned}
& \operatorname{Ext}_{C}^{2}\left(I_{3}, P_{1}\right) \cong k \quad, \quad \operatorname{Ext}_{C}^{2}\left(I_{3}, P_{3}\right) \cong k \\
& \operatorname{Ext}_{C}^{2}\left(I_{1}, P_{1}\right) \cong k \quad, \quad \operatorname{Ext}_{C}^{2}\left(I_{1}, P_{3}\right) \cong k .
\end{aligned}
$$

Since the projective dimension of $I_{2}$ is one and the injective dimension of $P_{2}$ is also one, this yields $\operatorname{dim}_{k} \operatorname{Ext}_{C}^{2}(D C, C)=4$. Using Lemma 2.7 we get the new indecomposable projectives

$\begin{array}{lll}1 & 3 & \\ 3 & 1 & 2 \\ 1 & 3 & \end{array}$.

Thus, a system of relations for the relation-extension algebra is $\alpha \beta=0, \delta \alpha=$ $0, \beta \delta=0$ and $\delta \gamma \delta=0$. 


\section{Cluster-tilted algebras}

\subsection{Preliminaries}

Let $A$ be a hereditary algebra. The cluster category $\mathcal{C}_{A}$ of $A$ is defined as follows. Let $F$ denote the automorphism of $\mathcal{D}^{b}(\bmod A)$ defined as the composition $\tau_{\mathcal{D}^{b}(\bmod A)}^{-1}[1]$, where $\tau_{\mathcal{D}^{b}(\bmod A)}^{-1}$ is the Auslander-Reiten translation in $\mathcal{D}^{b}(\bmod A)$, and [1] is the shift functor. Then $\mathcal{C}_{A}$ is the quotient category $\mathcal{D}^{b}(\bmod A) / F$. Its objects are the $F$-orbits $\tilde{X}=\left(F^{i} X\right)_{i \in \mathbb{Z}}$, where $X$ is an object in $\mathcal{D}^{b}(\bmod A)$. The set of morphisms from $\tilde{X}=\left(F^{i} X\right)_{i \in \mathbb{Z}}$ to $\tilde{Y}=\left(F^{i} Y\right)_{i \in \mathbb{Z}}$ in $\mathcal{C}_{A}$ is given by

$$
\operatorname{Hom}_{\mathcal{C}_{A}}(\tilde{X}, \tilde{Y})=\bigoplus_{i \in \mathbb{Z}} \operatorname{Hom}_{\mathcal{D}^{b}(\bmod A)}\left(X, F^{i} Y\right)
$$

It is shown in 20], that $\mathcal{C}_{A}$ is a triangulated category. Furthermore, the canonical functor $\mathcal{D}^{b}(\bmod A) \rightarrow \mathcal{C}_{A}$ is a functor of triangulated categories. We refer to [6] for facts about the cluster category.

An object $\tilde{T}$ in $\mathcal{C}_{A}$ is called a tilting object provided $\operatorname{Ext}_{\mathcal{C}_{A}}^{1}(\tilde{T}, \tilde{T})=0$ and the number of isomorphism classes of indecomposable summands of $\tilde{T}$ equals the number of isomorphism classes of simple $A$-modules (that is, the number of points in the quiver of $A$ ). The algebra of endomorphisms $\tilde{C}=\operatorname{End}_{\mathcal{C}_{A}}(\tilde{T})$ is then called a cluster-tilted algebra [7].

Cluster-tilted algebras may also be expressed in terms of modules. We recall that an $A$-module $T$ is called a tilting module provided $\operatorname{Ext}_{A}^{1}(T, T)=0$ and the number of isomorphism classes of indecomposable summands of $T$ equals the number of isomorphism classes of simple $A$-modules. Denoting by $\tilde{T}$ the $F$-orbit of $T$, we have the following theorem.

Theorem $3.1(([\underline{6}, 3.3]))$ Let $\tilde{C}$ be a cluster-tilted algebra, then there exist a hereditary algebra $A$ and a tilting $A$-module $T$ such that $\tilde{C} \cong \operatorname{End}_{\mathcal{C}_{A}}(\tilde{T})$.

We further recall that the endomorphism algebra of a tilting module over a hereditary algebra is called a tilted algebra, see, for instance, 22. We need the following result.

Theorem $3.2(([\mathbf{1 7}]))$ Let $A$ be a hereditary algebra, $T$ be a tilting A-module and $C=\operatorname{End}_{A}(T)$ be the corresponding tilted algebra. Then

(a) The derived functor $\operatorname{RHom}_{A}(T,-): \mathcal{D}^{b}(\bmod A) \rightarrow \mathcal{D}^{b}(\bmod C)$ is an equivalence of categories which maps the A-module $T$ to the $C$-module $C$.

(b) $\mathrm{RHom}_{A}(T,-)$ commutes with the Auslander-Reiten translations and the shifts in the respective categories.

\subsection{Cluster-tilted algebras are trivial extensions}

For any object $X$ in $\mathcal{D}^{b}(\bmod A)$, the $k$-vector space $\operatorname{Hom}_{\mathcal{D}^{b}(\bmod A)}(X, F X)$ has a natural structure of $\operatorname{End}_{\mathcal{D}^{b}(\bmod A)}(X)-\operatorname{End}_{\mathcal{D}^{b}(\bmod A)}(X)$-bimodule under the 
action

$$
\begin{aligned}
\operatorname{End}(X) \times \operatorname{Hom}(X, F X) \times \operatorname{End}(X) & \rightarrow \operatorname{Hom}(X, F X) \\
(u, f, v) & \mapsto F u \circ f \circ v
\end{aligned}
$$

The following lemma is proved in [3, 3.1]. We include a simple proof for the convenience of the reader.

Lemma 3.3 Let $\tilde{C}$ be a cluster tilted algebra. Then, for each hereditary algebra $A$ and tilting $A$-module $T$ such that $\tilde{C}=\operatorname{End}_{\mathcal{C}_{A}}(\tilde{T})$, we have

$$
\tilde{C} \cong \operatorname{End}_{A}(T) \ltimes \operatorname{Hom}_{\mathcal{D}^{b}(\bmod A)}(T, F T) .
$$

Proof. By definition of $\mathcal{C}_{A}$, we have

$$
\tilde{C}=\operatorname{End}_{\mathcal{C}_{A}}(\tilde{T})=\oplus_{i \in \mathbb{Z}} \operatorname{Hom}_{\mathcal{D}^{b}(\bmod A)}\left(T, F^{i} T\right)
$$

as $k$-vector spaces, and the multiplication is given by

$$
\left(g_{i}\right)_{i \in \mathbb{Z}}\left(f_{j}\right)_{j \in \mathbb{Z}}=\left(\sum_{i+j=l} F^{j} g_{i} \circ f_{j}\right)_{l \in \mathbb{Z}} .
$$

Since $A$ is hereditary, then, for any two $A$-modules $M$ and $N$, we have that $\operatorname{Hom}_{\mathcal{D}^{b}(\bmod A)}(M, N[i])=0$ for all $i \geq 2$. Therefore, as a $k$-vector space

$$
\tilde{C}=\operatorname{End}_{\mathcal{C}_{A}}(\tilde{T})=\operatorname{Hom}_{\mathcal{D}^{b}(\bmod A)}(T, T) \oplus \operatorname{Hom}_{\mathcal{D}^{b}(\bmod A)}(T, F T) .
$$

The multiplication of two elements $f, g \in \operatorname{End}_{\mathcal{C}_{A}}(\tilde{T})$ is given as follows. Assume $f=\left(f_{0}, f_{1}\right)$ and $g=\left(g_{0}, g_{1}\right)$, with $f_{0}, g_{0} \in \operatorname{Hom}_{\mathcal{D}^{b}(\bmod A)}(T, T)$ and $f_{1}, g_{1} \in$ $\operatorname{Hom}_{\mathcal{D}^{b}(\bmod A)}(T, F T)$, then, since $F g_{1} \circ f_{1}=0$,

$$
g f=\left(g_{0} \circ f_{0}, F g_{0} \circ f_{1}+f_{0} \circ g_{1}\right) .
$$

In view of the bimodule structure of $\operatorname{Hom}_{\mathcal{D}^{b}(\bmod A)}(T, F T)$ defined above, this shows indeed that $\tilde{C}=\operatorname{End}_{\mathcal{C}_{A}}(\tilde{T})$ is the trivial extension of $\operatorname{End}_{\mathcal{D}^{b}(\bmod A)}(T)=$ $\operatorname{End}_{A}(T)$ by the bimodule $\operatorname{Hom}_{\mathcal{D}^{b}(\bmod A)}(T, F T)$.

Since the algebra $\operatorname{End}_{A}(T)$ of the lemma is tilted, any cluster-tilted algebra is a trivial extension of a tilted algebra. However, the hereditary algebra $A$ and the $A$-module $T$ above are not unique. Therefore, one cannot apply directly the lemma to construct a map from cluster tilted algebras to tilted algebras.

\subsection{The main result}

We are now able to prove the main theorem of this section.

Theorem 3.4 An algebra $\tilde{C}$ is cluster-tilted if and only if there exists a tilted algebra $C$ such that $\tilde{C}$ is the relation-extension of $C$. 
Proof. Let $C$ be a tilted algebra. Then there exist a hereditary algebra $A$ and a tilting $A$-module $T$ such that $C=\operatorname{End}_{A}(T)$. Let $\tilde{T}$ denote as usual the $F$-orbit of $T$ in $\mathcal{D}^{b}(\bmod A)$. Then $\tilde{C}=\operatorname{End}_{\mathcal{C}_{A}}(\tilde{T})$ is a cluster-tilted algebra. By Lemma 3.3 we have

$$
\tilde{C}=\operatorname{End}_{\mathcal{D}^{b}(\bmod A)}(T) \ltimes \operatorname{Hom}_{\mathcal{D}^{b}(\bmod A)}(T, F T) .
$$

By Theorem 3.2 the derived functor $\mathrm{RHom}_{A}(T,-)$ induces $C$ - $C$-bimodule isomorphisms

$$
\operatorname{End}_{\mathcal{D}^{b}(\bmod A)}(T) \cong \operatorname{End}_{\mathcal{D}^{b}(\bmod C)}(C) \cong \operatorname{End}_{C}(C) \cong C
$$

and

$$
\operatorname{Hom}_{\mathcal{D}^{b}(\bmod A)}(T, F T) \cong \operatorname{Hom}_{\mathcal{D}^{b}(\bmod C)}\left(C, F^{\prime} C\right)
$$

where $F^{\prime}=\tau_{\mathcal{D}^{b}(\bmod C)}^{-1}[1]$ is the functor corresponding to $F$ in the derived category $\mathcal{D}^{b}(\bmod C)$. Thus we get

$$
\tilde{C} \cong C \ltimes \operatorname{Hom}_{\mathcal{D}^{b}(\bmod C)}\left(C, F^{\prime} C\right) .
$$

Moreover, we have the following sequence of $C$ - $C$-bimodule isomorphisms

$$
\begin{aligned}
\operatorname{Hom}_{\mathcal{D}^{b}(\bmod C)}\left(C, F^{\prime} C\right) & \cong \operatorname{Hom}_{\mathcal{D}^{b}(\bmod C)}\left(\tau_{\mathcal{D}^{b}(\bmod C)} C[1], C[2]\right) \\
& \cong \operatorname{Hom}_{\mathcal{D}^{b}(\bmod C)}(D C, C[2]) \\
& \cong \operatorname{Ext}_{C}^{2}(D C, C),
\end{aligned}
$$

where the first is obtained by applying to both arguments the automorphism $\tau_{\mathcal{D}^{b}(\bmod C)}[1]$, the second uses the fact that $\tau_{\mathcal{D}^{b}(\bmod C)} C \cong D C[-1]$ and the third is a property of the derived category. This shows that the relation-extension $C \ltimes \operatorname{Ext}_{C}^{2}(D C, C)$ is a cluster-tilted algebra. Finally, by Lemma 3.3 every cluster-tilted algebra is obtained in this way.

\subsection{Remarks and examples}

(a) Since the quiver of a tilted algebra has no oriented cycles, it follows directly from Theorem 3.4 and Theorem 2.6 that we have a construction for the quiver of a cluster-tilted algebra $\tilde{C}$ starting from the quiver of a tilted algebra $C$. This construction is easily seen to generalise the one in [11, 4.1] and, thus, can be used to relate the Happel-Vossieck list of tame concealed algebras [18] with Seven's list of minimal infinite cluster quivers [23.

(b) A different description, inspired from [19, of the relation-extension algebra is sometimes useful. Consider the following doubly infinite matrix algebra

$$
\hat{C}=\left[\begin{array}{ccccc}
\ddots & & & 0 & \\
& C_{i-1} & & & \\
& M_{i} & C_{i} & & \\
& & M_{i+1} & C_{i+1} & \\
& 0 & & & \ddots
\end{array}\right]
$$


where matrices are assumed to have only finitely many non-zero coefficients, $C_{i}=C$ and $M_{i}=\operatorname{Ext}_{C}^{2}(D C, C)$ for all $i \in \mathbb{Z}$, all the remaining coefficients are zero. The addition is the usual addition of matrices while the multiplication is induced from the bimodule structure of $\operatorname{Ext}_{C}^{2}(D C, C)$ and the zero map $\operatorname{Ext}_{C}^{2}(D C, C) \otimes_{C} \operatorname{Ext}_{C}^{2}(D C, C) \rightarrow 0$. Clearly, $\hat{C}$ is a Galois covering of $C \ltimes \operatorname{Ext}_{C}^{2}(D C, C)$ with group $\mathbb{Z}$ : the identity maps $C_{i} \rightarrow C_{i+1}$, $M_{i} \rightarrow M_{i+1}$ induce an automorphism $\eta$ of $\hat{C}$ and $\hat{C} / \eta \cong C \ltimes \operatorname{Ext}_{C}^{2}(D C, C)$.

(c) As observed before, different tilted algebras $C$ may correspond to the same cluster-tilted algebra $\tilde{C}$ (thus, the surjective map $C \mapsto \tilde{C}$ is not injective). We give an example of such an occurrence.

Example 3.5 Let $C_{1}$ be given by the quiver

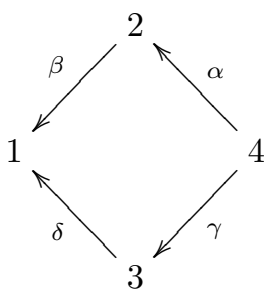

bound by $\alpha \beta=\gamma \delta$. This is a tilted algebra of Dynkin type $D_{4}$, and the corresponding cluster-tilted (relation-extension) algebra $\tilde{C}_{1}$ is given by the quiver

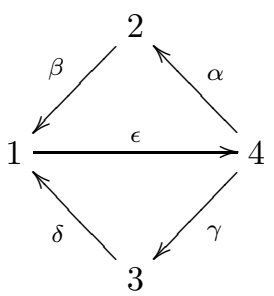

bound by $\alpha \beta=\gamma \delta, \beta \epsilon=0, \delta \epsilon=0, \epsilon \alpha=0, \epsilon \gamma=0$. Let now $C_{2}$ be the tilted algebra given by the quiver

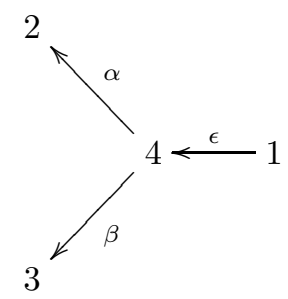

bound by $\epsilon \alpha=0, \epsilon \beta=0$. Then it is easily seen that $\tilde{C}_{1}=\tilde{C}_{2}$.

(d) Not surprisingly, it is possible that $C$ is representation-finite whereas $\tilde{C}$ is representation-infinite: it suffices to have two points $x, y \in\left(Q_{C}\right)_{0}$ such that $\operatorname{dim}_{k} \operatorname{Ext}_{C}^{2}\left(I_{y}, P_{x}\right)>1$. We give an example of such a situation. 
Example 3.6 Let $C$ be given by the quiver

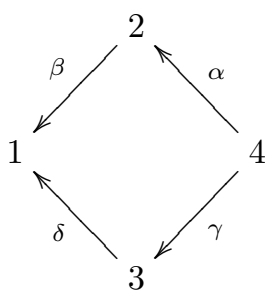

bound by $\alpha \beta=0, \gamma \delta=0$. This is a representation-finite tilted algebra of euclidean type $\tilde{A}_{3}$. The injective resolution

$$
0 \rightarrow P_{1} \rightarrow I_{1} \rightarrow I_{2} \oplus I_{3} \rightarrow I_{4} \oplus I_{4} \rightarrow 0
$$

shows that $\operatorname{dim}_{k} \operatorname{Ext}_{C}^{2}\left(I_{4}, P_{1}\right)=2$. The corresponding cluster-tilted algebra $\tilde{C}$ is given by the quiver

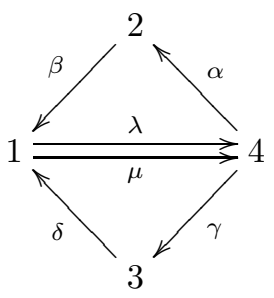

bound by $\alpha \beta=0, \gamma \delta=0, \delta \lambda=0, \lambda \gamma=0, \beta \mu=0, \mu \alpha=0$. The indecomposable projective $\tilde{C}$-modules are given by

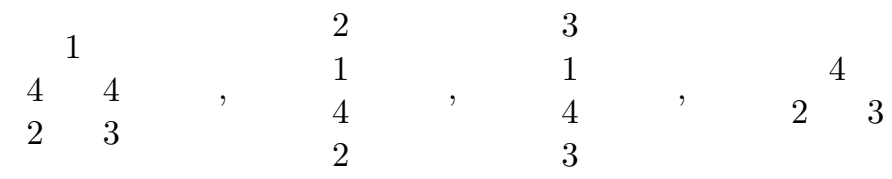

Clearly, $\tilde{C}$ is representation-infinite.

(e) The relation-extension algebra in Example 2.8 is not a cluster-tilted algebra. This follows from the fact that cluster-tilted algebras contain no oriented cycles of length two.

\section{References}

[1] I. Assem N. Marmaridis, 'Tilting modules over split-by nilpotent extensions', Comm. Algebra 26 (1998) 1547-1555.

[2] M. Auslander, I. Reiten S.O. Smalø, Representation Theory of Artin Algebras Cambridge Studies in Advanced Math. 36, (Cambridge University Press, Cambridge, 1995). 
[3] Z. Bin, 'Equivalences between cluster categories', preprint 2005, arXiv:math.RT/0511382

[4] K. Bongartz, 'Algebras and quadratic forms', J. London Math. Soc. (2), 28 (1983), 461-469.

[5] K. Bongartz P. Gabriel, 'Covering spaces in representation theory', Invent. Math., 65 (1981/82), No.3, 331-378.

[6] A. B. Buan, R. Marsh, M. Reineke, I. Reiten G. Todorov, 'Tilting theory and cluster combinatorics', to appear in Adv. Math.

[7] A. B. Buan, R. Marsh I. Reiten, 'Cluster-tilted algebras', to appear in Trans. Amer. Math. Soc.

[8] A. B. Buan, R. Marsh I. Reiten, 'Cluster mutation via quiver representations', preprint 2004, arXiv:math.RT/0412077

[9] A. B. Buan, R. Marsh I. Reiten, 'Cluster-tilted algebras of finite representation type', preprint 2005, arXiv:math.RT/0509198.

[10] A. B. Buan I. Reiten, 'From tilted to cluster-tilted algebras of Dynkin type', preprint 2005, arXiv:math.RT/0510445

[11] A. B. Buan, I. Reiten A. I. Seven, 'Tame concealed algebras and cluster algebras of minimal infinite type', preprint 2005, arXiv:math.RT/0512137

[12] P. Caldero, F. Chapoton R. Schiffler, 'Quivers with relations arising from clusters $\left(A_{n}\right.$ case)', Trans. Amer. Math. Soc. 358 (2006), no. 3, 1347-1364.

[13] P. Caldero, F. Chapoton R. Schiffler, 'Quivers with relations and cluster tilted algebras', to appear in Algebras and Representation Theory.

[14] S. Fomin A. Zelevinsky, 'Cluster algebras I. Foundations', J. Amer. Math. Soc. $15(2)$ (2002) 497-529 (electronic)

[15] S. Fomin A. Zelevinsky, 'Cluster algebras II. Finite type classification', Inventiones Mathematicae 154(1) (2003) 63-121.

[16] R. M. Fossum, P. A. Griffith I. Reiten, Trivial extensions of abelian categories, Lecture Notes in Math. 456, (Springer, Berlin, New York, 1975).

[17] D. Happel, Triangulated Categories in the Representation Theory of Finite Dimensional Algebras, London Mathematical Society. Lecture Notes Series 119, (Cambridge University Press, Cambridge 1988).

[18] D. Happel D. Vossieck, 'Minimal algebras of infinite representation type with preprojective component', Manuscripta Math., 42 (1983) 221-243.

[19] D. Hughes J. Waschbüsch, 'Trivial extensions of tilted algebras', Proc. London Math. Soc. 46 (1983) 347-364. 
[20] B. Keller, 'On triangulated orbit categories', Documenta Math. 10 (2005), 551-581.

[21] B. Keller I. Reiten, 'Cluster-tilted algebras are Gorenstein and stably Calabi-Yau', preprint (2005), arXiv:math.RT/0512471

[22] C. M. Ringel, Tame algebras and integral quadratic forms, Lecture Notes in Math. 1099, (Springer, Berlin, 1984).

[23] A. I. Seven, 'Recognizing cluster algebras of finite type', preprint 2004, arXiv:math. CO/0406545.

I. Assem

Département de Mathématiques,

Université de Sherbrooke,

Sherbrooke (Québec), J1K 2R1, Canada

ibrahim.assem@usherbrooke.ca

T. Brüstle

Département de Mathématiques,

Université de Sherbrooke,

Sherbrooke (Québec), J1K 2R1 Canada

thomas.brustle@usherbrooke.ca

and

Department of Mathematics,

Bishop's University,

Lennoxville, (Québec), J1M 1Z7, Canada

tbruestl@ubishops.ca

R. Schiffler

Department of Mathematics and Statistics, University of Massachusetts at Amherst, Amherst, MA 01003-9305, USA

schiffler@math.umass.edu 